\title{
Synthesis and Mesogenic Properties of Lipophilic and Amphiphilic Tetraphenylporphyrins
}

\author{
Natal'ya A. Bragina, ${ }_{1}{ }^{\text {Irina N. Fedulova }}{ }_{1}{ }^{a}$ Nickita V. Novikov, ${ }^{a}$ \\ Ekaterina S. Krutikova, ${ }^{a}$ Andrey F. Mironov, ${ }^{a}{ }^{@ 1}$ Venera V. Bykova, $^{\text {b }}$ \\ Galina A. Ananieva, ${ }^{\text {b }}$ and Nadezhda V. Usol'tseva ${ }^{\mathrm{b} 2}$ \\ ${ }^{a}$ M.V. Lomonosov Moscow State Academy of Fine Chemical Technology, Moscow, 119571, Russia \\ ${ }^{b}$ Research Institute of Nanomaterials, Ivanovo State University, Ivanovo, 153025, Russia \\ ${ }^{\circledR 1}$ Corresponding author E-mail: mironov@mitht.ru \\ ${ }^{\circledR 2}$ Corresponding authorE-mail: usol@ivanovo.ac.ru
}

Liquid crystals, based on porphyrins, belong to the discotic mesogenes and are of considerable interest for use in optoelectronics and devices for display and storage of information. Porphyrins possess all the necessary properties for that, including synthetic versatility, thermal stability and a number of special photochemical properties. In comparison with widely studied class of phthalocyanines liquid crystals based on porphyrins have lower melting points. In this study we have synthesized homologous series of lipophilic symmetrical 5,15-di-and 5,10,15,20-tetrameso-arylsubstituted porphyrins containing long chain alkyl and acyl groups, their zinc and cobalt complexes, amphiphilic derivatives and studied their mesogenic properties. The series of 5,15-di-and 5,10,15,20-tetra-mesoarylsubstituted porphyrins differ by the quantity and the length of peripherical aliphatic substitutents, type of their attachment to the porphyrin meso-phenyl groups, nature of terminal group and presence of a metal. The chain length of lipophilic substituents was varied from $C_{8}$ to $C_{18}$ atoms. Studying of mesogenic properties has allowed us to reveal and generalize structural influence on the ability of porphyrins to form mesophases. We've used efficient synthetic procedures to obtain these meso-arylsubstituted porphyrins. We have suggested the new approach to the synthesis of the lipoporphyrins. According to this approach the residues of higher fatty acids and alcohols are incorporated into the molecules of benzaldehyde at the initial stages of synthesis which is followed by condensation with pyrrole. This method essentially simplifies the synthetic procedure, facilitates the purification of the target porphyrins, gives the chance to carry out synthesis in soft conditions and provides higher yields of lipoporphyrins. Synthesis of 5,15-dimeso-arylsubstituted porphyrins was carried out using dipyrrolylmethanes in 36-44\% yields. 5,10,15,20-Tetra-mesoarylsubstituted porphyrins were synthesised by monopyrrole condensation in 33-45\% yields. Amphiphilic derivatives were prepared by chemical modification of periferical aliphatic residues. Zinc and cobalt complexes were obtained in 90-95\% yields from porphyrin ligands. The mesomorphic behavior of the porphyrin derivatives was investigated by using optical polarizing microscopy and differential scanning calorimetry. The phase transition temperatures of all compounds in the course of heating are summarized. It has been shown that eight compounds among long chain meso-arylsubstituted porphyrins could form mesophase and exhibit thermotropic and lyotropic behaviour in nonpolar solvents. Five compounds in the series of 5,15-disubstituted and one compound among 5,10,15,20-tetra-mesoarylsubstituted porphyrins were amphotropic: the thermotropic and lyotropic mesomorphism in nonpolar solvents were observed. Two 5,10,15,20-tetra-meso-arylsubstituted porphyrins exhibited only thermotropic mesomorphism. Seven porphyrins derivatives were glassing mesogens. The mesophase in all compounds was characterized by nongeometric grain structure presumably associated with a column-type packing. As a result it has been established that discotic symmetrical long chain meso-arylsubstituted porphyrins exhibit thermotropic and lyotropic behaviour in nonpolar solvents, some of them are glassing mesogens. It is obvious, the mesophase range and glassing properties of these porphyrins are influenced, first of all, by the quantity and the chain length of lipophilic substituents, and also by the type of their attachment to the porphyrin meso-phenyl groups. We have shown, that replacement of donor alkoxy by acceptor alkanoyloxy groups influences on mesogenic properties of the porphyrins derivatives. Incorporation of coordinatively unsaturated metals $\left(\mathrm{Zn}^{I I}\right.$ and $\mathrm{Co}^{I I}$ ) increases the number of mesogenic porphyrins derivatives in the series of 5,15-disubstituted porphyrins and considerably changes mesogenic properties in comparison with corresponding ligands. It has been shown, that liquid crystalline properties in the case of 5,15-disubstituted porphyrins with equal lengths of aliphatic substituents at the meso-positions and the corresponding metal complexes are more similar. Thus, we have expanded databank of discotic liquid crystals based on lipophilic porphyrins derivatives and their metal complexes. The mesomorphic properties of porphyrins indicate that they offer potential usage in optoelectronic field.

Keywords: meso-Arylsubstituted porphyrins, metallocomplexes, liquid crystals, discotic mesogenes, mesophase, thermotropic and lyotropic mesomorphism, glassing. 


\title{
Синтез и мезогенные свойства липофильных и амфифильных тетрафенилпорфиринов
}

\author{
Н.А. Брагина, ${ }^{a}$ И.Н. Федулова, ${ }^{a}$ Н.В. Новиков, ${ }^{a}$ Е.С. Крутикова, ${ }^{a}$ А.Ф. Миронов, ${ }^{a} 1$ \\ В.В. Быкова, ${ }^{\mathrm{b}}$ Г.А. Ананьева, ${ }^{\mathrm{b}}$ H.В. Усольцева ${ }^{\mathrm{b}} 2$
}

${ }^{a}$ Московская государственная академия тонкой химической технологии им. М.В. Ломоносова, Москва, 119571, Россия

${ }^{b}$ Ивановский государственный университет, Иваново, 153025, Россия

${ }^{\circledR 1}$ E-mail:mironov@mitht.ru

${ }^{@ 2}$ E-mail:usol@ivanovo.ac.ru

В работе сообщается о синтезе мезогенных 5,15- и 5,10,15,20-мезо-арилзамещенных порфиринов и их металлокомплексов, которые содержат липофильные алкильные и ацильные заместители с длиной цуепи от $C_{8}$ до $C_{18}$ атомов. Жидкокристаллические свойства полученных порфиринов изучали методами оптической поляризационной микроскопии и дифференциальной сканирующей калориметрии. Выявлень мезо-арилзамещенные порфирины, проявляющие термотропный мезоморфизм и лиотропный мезоморфизм в неполярных растворителях, а также соединения, способные стекловаться с сохранением структуры мезофазы.

Ключевые слова: мезо-Арилзамещенные порфирины, металлокомплексы, жидкие кристаллы, дискотические мезогены, мезофаза, термотропный, лиотропный мезоморфизм, стеклование.

\section{Введение}

Тетрафенилпорфирин (ТФП) и его производные являются перспективными объектами для создания самоорганизующихся супрамолекулярных структур и представляют интерес для создания новых материалов для нелинейной оптики, катализа, сенсорных систем, медицины и др. ${ }^{[1,2]}$ В последние годы возрастает интерес к порфиринам как к потенциальным лиотропным и термотропным дискотическим мезогенам. ${ }^{[3]}$ Жидкие кристаллы (ЖК) на основе порфиринов и родственных тетрапиррольных соединений (фталоцианинов, тетраазапорфиринов) могут найти практическое применение в области оптоэлектроники (светоизлучающие приборы) и устройств отображения и хранения информации. ${ }^{[4-6]}$ Порфирины обладают всеми необходимыми для этого качествами, включая доступ- ность исходных соединений, легкость синтеза и функционализации, термическую устойчивость и ряд особых фотохимических свойств. По сравнению с широко изучаемым классом фталоцианинов жидкие кристаллы на основе порфиринов имеют более низкую точку плавления. ${ }^{[3]}$

Первый пример термотропного мезоморфизма порфиринов был описан в 1980 г., когда был синтезирован замещенный бис-гидрохлорид уропорфирина с монотропной мезофазой, существовавшей в очень узком диапазоне $0,1^{\circ} \mathrm{C} .{ }^{[7]} \mathrm{B}$ настоящее время успешно решается задача направленного синтеза мезоморфных производных порфиринов, ${ }^{[8-12]}$ и в связи с этим можно выделить три структурных типа порфиринов, проявляющих ЖК свойства: $\quad \beta$-замещенные октаалкилпорфирины ${ }^{[13-14]}$ (Тип 1), 5,15-дифенилпорфирины ${ }^{[15-17]}$ (Тип 2), мезоарилзамещенные ТФП ${ }^{[8,9,12]}$ (Тип 3 ) (Рисунок 1).

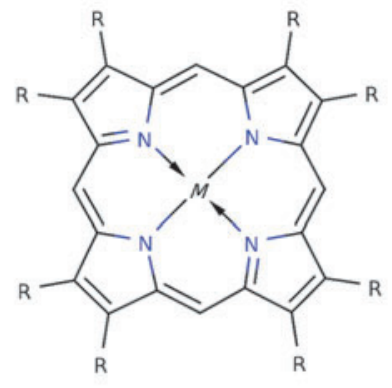

Тип 1

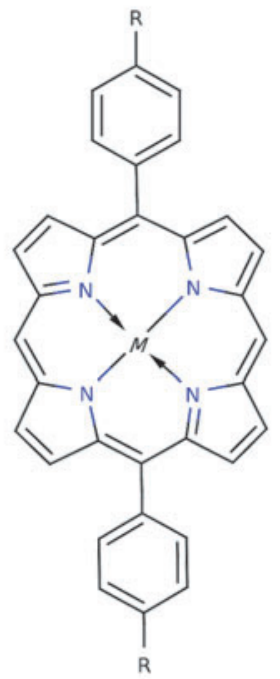

Тй 2

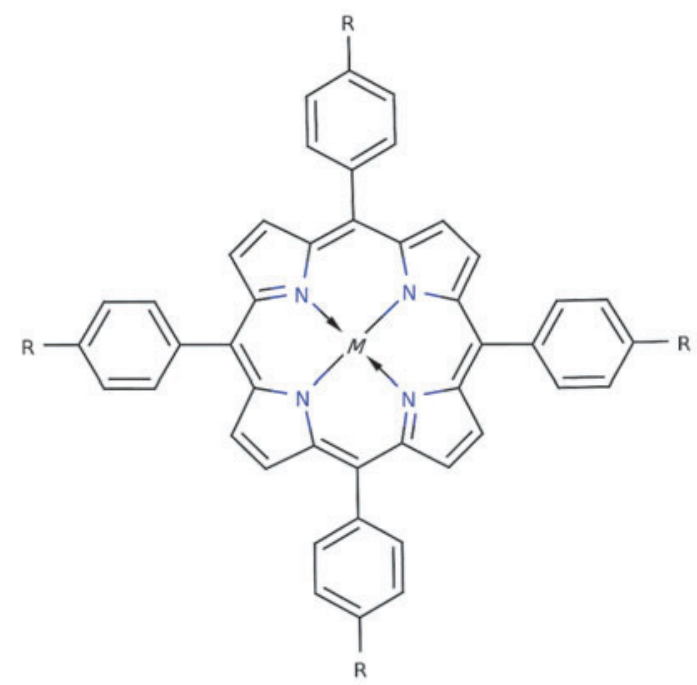

ТиII 3

Рисунок 1. Структурные типы мезогенных порфиринов. 
Многообразия данных молекулярных структур можно достигнуть за счет изменения протяженности заместителей, их разветвления, изменения их позиций и количества, включения в алифатическую цепь гетероатомов и мостиковых групп, вариаций металлакомплексообразователя и «жесткого» фрагмента молекулы, синтеза ди- и тримеров и т.д. ${ }^{[3]}$ Однако, несмотря на наличие публикаций по мезоморфизму порфиринов-лигандов ${ }^{[8-10]}$ и комплексов порфиринов с металлами, ${ }^{[11,18-20]}$ в том числе лантаноидами, систематические исследования взаимосвязи между структурой порфиринов и их способностью к мезоморфизму в настоящее время отсутствуют.
Целью данной работы явился синтез симметричных липофильных мезо-арилзамещенных порфиринов с протяженными гидрофобными заместителями и их металлокомплексов для выявления мезогенных соединений и установления структурно-функциональных зависимостей.

\section{Экспериментальная часть}

5,15-Дизамещенные порфирины 1-6 (Таблица 1) получали с выходами 36-44 \% исходя из соответствующих дипирролилметановибензальдегида, согласноранееописанным методам. ${ }^{[21,22]}$ 5,10,15,20-Тетразамещенные порфирины 7-17

Таблица 1. Структура 5,15-дизамещенных порфиринов.

\begin{tabular}{|c|c|c|c|}
\hline Структура соединения & № & $\mathrm{R}$ & M \\
\hline & 1 & $-\mathrm{O}\left(\mathrm{CH}_{2}\right)_{7} \mathrm{CH}_{3}$ & $2 \mathrm{H}$ \\
\hline & $1 \mathrm{a}$ & $-\mathrm{O}\left(\mathrm{CH}_{2}\right)_{7} \mathrm{CH}_{3}$ & $\mathrm{Zn}^{\mathrm{II}}$ \\
\hline & 10 & $-\mathrm{O}\left(\mathrm{CH}_{2}\right)_{7} \mathrm{CH}_{3}$ & $\mathrm{Co}^{\mathrm{II}}$ \\
\hline & 2 & $-\mathrm{O}\left(\mathrm{CH}_{2}\right)_{13} \mathrm{CH}_{3}$ & $2 \mathrm{H}$ \\
\hline & 3 & $-\mathrm{O}\left(\mathrm{CH}_{2}\right)_{15} \mathrm{CH}_{3}$ & $2 \mathrm{H}$ \\
\hline & 4 & $-\mathrm{O}\left(\mathrm{CH}_{2}\right)_{17} \mathrm{CH}_{3}$ & $2 \mathrm{H}$ \\
\hline & 5 & $-\mathrm{O}\left(\mathrm{CH}_{2}\right)_{5} \mathrm{COOH}$ & $2 \mathrm{H}$ \\
\hline R & 6 & $-\mathrm{O}\left(\mathrm{CH}_{2}\right)_{10} \mathrm{COOH}$ & $2 \mathrm{H}$ \\
\hline
\end{tabular}

Таблица 2. Структура 5,10,15,20-тетразамещенных порфиринов.

\begin{tabular}{|c|c|c|c|}
\hline Структура соединения & № & $\mathrm{R}$ & $\mathrm{M}$ \\
\hline & 7 & $\mathrm{O}\left(\mathrm{CH}_{2}\right)_{7} \mathrm{CH}_{3}$ & $2 \mathrm{H}$ \\
\hline & $7 \mathbf{a}$ & $\mathrm{O}\left(\mathrm{CH}_{2}\right)_{7} \mathrm{CH}_{3}$ & $\mathrm{Zn}^{\mathrm{II}}$ \\
\hline & 70 & $\mathrm{O}\left(\mathrm{CH}_{2}\right)_{7} \mathrm{CH}_{3}$ & $\mathrm{Co}^{\mathrm{II}}$ \\
\hline & 8 & $\mathrm{O}\left(\mathrm{CH}_{2}\right)_{9} \mathrm{CH}_{3}$ & $2 \mathrm{H}$ \\
\hline & $8 \mathbf{a}$ & $\mathrm{O}\left(\mathrm{CH}_{2}\right)_{9} \mathrm{CH}_{3}$ & $\mathrm{Zn}^{\mathrm{II}}$ \\
\hline & 86 & $\mathrm{O}\left(\mathrm{CH}_{2}\right)_{9} \mathrm{CH}_{3}$ & $\mathrm{Co}^{\mathrm{II}}$ \\
\hline $\mathbf{R}$ & 9 & $\mathrm{O}\left(\mathrm{CH}_{2}\right)_{13} \mathrm{CH}_{3}$ & $2 \mathrm{H}$ \\
\hline & 9a & $\mathrm{O}\left(\mathrm{CH}_{2}\right)_{13} \mathrm{CH}_{3}$ & $\mathrm{Zn}^{\mathrm{II}}$ \\
\hline & 96 & $\mathrm{O}\left(\mathrm{CH}_{2}\right)_{13} \mathrm{CH}_{3}$ & $\mathrm{Co}^{\mathrm{II}}$ \\
\hline & 10 & $\mathrm{O}\left(\mathrm{CH}_{2}\right)_{15} \mathrm{CH}_{3}$ & $2 \mathrm{H}$ \\
\hline & $10 \mathrm{a}$ & $\mathrm{O}\left(\mathrm{CH}_{2}\right)_{15} \mathrm{CH}_{3}$ & $\mathrm{Zn}^{\mathrm{II}}$ \\
\hline & $10 \sigma$ & $\mathrm{O}\left(\mathrm{CH}_{2}\right)_{15} \mathrm{CH}_{3}$ & $\mathrm{Co}^{\mathrm{II}}$ \\
\hline & 11 & $\mathrm{O}\left(\mathrm{CH}_{2}\right)_{17} \mathrm{CH}_{3}$ & $2 \mathrm{H}$ \\
\hline & $11 \mathrm{a}$ & $\mathrm{O}\left(\mathrm{CH}_{2}\right)_{17} \mathrm{CH}_{3}$ & $\mathrm{Zn}^{\mathrm{II}}$ \\
\hline & $11 \sigma$ & $\mathrm{O}\left(\mathrm{CH}_{2}\right)_{17} \mathrm{CH}_{3}$ & $\mathrm{Co}^{\mathrm{II}}$ \\
\hline & 12 & $\mathrm{OCO}\left(\mathrm{CH}_{2}\right)_{6} \mathrm{CH}_{3}$ & $2 \mathrm{H}$ \\
\hline & 13 & $\mathrm{OCO}\left(\mathrm{CH}_{2}\right)_{10} \mathrm{CH}_{3}$ & $2 \mathrm{H}$ \\
\hline & $13 a$ & $\mathrm{OCO}\left(\mathrm{CH}_{2}\right)_{10} \mathrm{CH}_{3}$ & $\mathrm{Zn}^{\mathrm{II}}$ \\
\hline $\mathbf{R}$ & $13 \sigma$ & $\mathrm{OCO}\left(\mathrm{CH}_{2}\right)_{10} \mathrm{CH}_{3}$ & $\mathrm{Co}^{\mathrm{II}}$ \\
\hline & 14 & $\mathrm{OCO}\left(\mathrm{CH}_{2}\right)_{12} \mathrm{CH}_{3}$ & $2 \mathrm{H}$ \\
\hline & $14 a$ & $\mathrm{OCO}\left(\mathrm{CH}_{2}\right)_{12} \mathrm{CH}_{3}$ & $\mathrm{Zn}^{\mathrm{II}}$ \\
\hline & 140 & $\mathrm{OCO}\left(\mathrm{CH}_{2}\right)_{12} \mathrm{CH}_{3}$ & $\mathrm{Co}^{\mathrm{II}}$ \\
\hline & 15 & $\mathrm{OCO}\left(\mathrm{CH}_{2}\right)_{14} \mathrm{CH}_{3}$ & $2 \mathrm{H}$ \\
\hline & 16 & $\mathrm{OCO}\left(\mathrm{CH}_{2}\right)_{5} \mathrm{Br}$ & $\mathrm{H}$ \\
\hline & 17 & $\mathrm{OCO}\left(\mathrm{CH}_{2}\right)_{10} \mathrm{Br}$ & $\mathrm{H}$ \\
\hline
\end{tabular}


(Таблица 2) получали по методу Линдсея ${ }^{[23]}$ в мягких условиях в среде хлористого метилена из соответствующих замещенных бензальдегидов и пиррола с выходами $33-45$ \%, как описано в работе ${ }^{[22]}$. Амфифильные производные порфиринов 5, 6 получали исходя из порфиринов-предшественников путем щелочного гидролиза терминальных карбоксиметильных групп в смеси тетрагидрофуран-вода. ${ }^{[24]}$ Металлокомплексы порфиринов с цинком и кобальтом были получены с выходами 90-95\% по стандартным методикам с использованием соответствующих ацетатов в смеси хлороформ-метанол. ${ }^{[16,25]}$

Индивидуальность и структуру полученных соединений подтверждали методами ТCX, ${ }^{1} \mathrm{H}$ ЯМР, масс-спектрометрии и даннымиэлементногоанализа, их спектральные характеристики приведены в работах ${ }^{[12,22,24]}$ и соответствуют литературным данным. ${ }^{[26-27]}$ Электронные спектры поглощения снимали на спектрофотометре Jasko UV-7800 в хлористом метилене. ИКспектры записывали на фурье-спектрометре Bruker EQUINOX 55 (Германия) в тонком слое или таблетке с $\mathrm{KBr}$. Спектры ЯМР регистрировали на импульсном фурье-спектрометре Bruker MSL-300 (Германия) с рабочей частотой на ядрах ${ }^{1} \mathrm{H}$ 300.13 МГц. Измерения химических сдвигов проводили по шкале $\delta$, внутренний стандарт $\mathrm{TMC}$, растворители $\mathrm{CDCl}_{3}$, $\mathrm{CD}_{3} \mathrm{OD}$. Масс-спектры снимали на приборе Bruker Ultraflex TOF/TOF (Германия) методом MALDI. Элементный анализ выполняли на С,H,N,S-элементном анализаторе Thermo Finnigan (Италия). ТCX осуществляли на пластинках Kieselgel 254 фирмы “Merck” (Германия). Хроматографическую очистку соединений проводили на открытых колонках с силикагелем G 60 (Sigma).

Мезоморфные свойства порфиринов 1-17 исследовали методом оптической поляризационной микроскопии. Термотропный мезоморфизм изучали, используя оптический термополяризационный микроскоп типа Leitz Laborlux 12 Pol, снабженный термостоликом Mettler FP 82. Лиотропный мезоморфизм исследовали методом контактных препаратов с толуолом, бензолом, хлороформом. Методом дифференциальной сканирующей калориметрии (ДСК) изучали фазовые переходы на приборе DSC 200 PC Phox (фирмы Netzsch). Измерения проводили в интервале температур от -30 до $85^{\circ} \mathrm{C}$ со скоростью $10^{\circ} \mathrm{C} /$ мин.

\section{Результаты и обсуждение}

В ходе исследования нами были синтезированы несколько групп липофильных 5,15-дизамещенных и 5,10,15,20-тетразамещенных порфиринов, их металлокомплексы, амфифильные производные и изучены их мезогенные свойства. Полученные результаты дополнили имеющиеся у нас данные по мезоморфизму липофильных мезо-арилзамещенных порфиринов, ${ }^{[12,17]}$ позволили нам выявить и обобщить некоторые особенности влияния элементов структуры порфиринов на способность к формированию мезофаз.

$$
\text { Представленные серии 5,15-дизамещенных }
$$

(1-6), 5,10,15,20-тетразамещенных (7-11 и 12-17) симметричных липофильных мезо-арилзамещенных порфиринов различаются количеством и протяженностью периферийных алифатических заместителей, способом их присоединения к фенильным остаткам порфирина и природой терминальной группы, наличием металла-комплексообразователя. Основные пути осуществленной нами структурной модификации ТФП схематически показаны на Рисунке 2. Ранее было установлено, что протяженность заместителей играет значительную роль в проявлении подобными соединениями мезоморфных свойств и формировании определенного типа мезофаз. ${ }^{[3,18]}$ Сочетание жесткого порфиринового макроцикла и гибких длинноцепных заместителей предопределяет тенденцию молекул к преимущественно параллельному расположению относительно друг друга. Функционализация порфирина длинноцепочечными алкильными заместителями, помимо придания ЖК-свойств, улучшает их растворимость в неполярных растворителях и способствует поверхностной иммобилизации. ${ }^{[20]}$

Для получения соединений 1-17 в препаративных количествах мы использовали эффективные методы синтеза мезо-арилзамещенных порфиринов. Ранее мы предложили новый подход к синтезу данных липопорфиринов, согласно которому остатки высших жирных кислот и спиртов вводят в производные бензальдегида на начальных стадиях синтеза, а затем проводят их конденсацию с пирролом. ${ }^{[22]}$ Это существенно сокращает и упрощает схему синтеза, позволяет избежать проблем, которые связаны с низкой растворимостью промежуточных гидроксифенилпорфиринов, облегчает очистку целевых продуктов, дает возможность осуществлять синтез в мягких условиях и получать липопорфирины с более высокими выходами. Синтез 5,15-дизамещенных

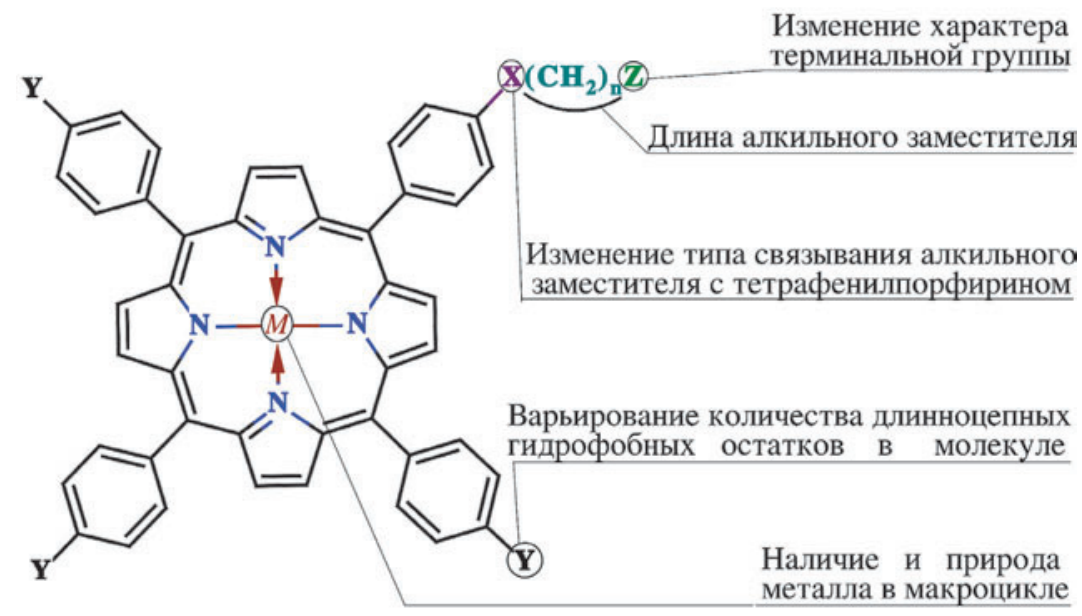

Рисунок 2. Основные модификации структуры мезо-арилзамещенных порфиринов. 
Таблица 3. Данные по изучению термотропного и лиотропного мезоморфизма порфиринов (1-17).

\begin{tabular}{|c|c|c|}
\hline № & $\begin{array}{c}\text { Температуры фазовых } \\
\text { переходов, }{ }^{\circ} \mathrm{C}\end{array}$ & $\begin{array}{l}\text { Лиотропный } \\
\text { мезоморфизм }\end{array}$ \\
\hline 1 & $\mathrm{Cr} \bullet 242.7 \mathrm{I}$ & - \\
\hline $1 \mathrm{a}$ & $\begin{array}{c}\mathrm{Cr} \bullet 131.4 \mathrm{Mes} \bullet 261.0 \mathrm{I} \\
\mathrm{I} \bullet 236.2 \mathrm{Mes} \bullet 115.0 \mathrm{G}\end{array}$ & $\begin{array}{c}+ \\
\text { (толуол, бензол, хлф.) }\end{array}$ \\
\hline 16 & $\begin{array}{c}\mathrm{Cr} \bullet 214.4 \mathrm{Mes} \bullet 290.2 \mathrm{I} \\
\mathrm{I} \bullet 157.2 \mathrm{Mes} \bullet 113.1 \mathrm{G}\end{array}$ & $\begin{array}{c}+ \\
\text { (толуол, бензол, хлф.) }\end{array}$ \\
\hline 2 & $\begin{array}{c}\mathrm{Cr} \bullet 72.8 \mathrm{Mes} \bullet 123.4 \mathrm{I} \\
\mathrm{I} \bullet 75.1 \mathrm{Mes} \bullet 48.2 \mathrm{G}\end{array}$ & $\begin{array}{c}+ \\
\text { (толуол, бензол, хлф.) }\end{array}$ \\
\hline 3 & $\begin{array}{l}\mathrm{Cr} \bullet 61.8 \mathrm{Mes} \bullet 87.7 \mathrm{I} \\
\mathrm{I} \bullet 36.4 \mathrm{Mes} \bullet 20.0 \mathrm{G}\end{array}$ & $\begin{array}{c}+ \\
\text { (толуол, бензол, хлф.) }\end{array}$ \\
\hline 4 & $\begin{array}{c}\mathrm{Cr} \bullet 120.0 \mathrm{I} \\
\mathrm{I} \bullet 40.1 \mathrm{Mes} \bullet 29.9 \mathrm{G}\end{array}$ & $\begin{array}{c}+ \\
\text { (толуол, бензол, хлф.) }\end{array}$ \\
\hline 5 & $\mathrm{Cr} \bullet 177.9 \mathrm{Mes} \bullet 232.5 \mathrm{I}$ & - \\
\hline 6 & $\mathrm{Cr} \bullet 233.2 \mathrm{I}$ & - \\
\hline 7 & $\mathrm{Cr} \bullet 209.6 \mathrm{I}$ & - \\
\hline $7 a$ & $\mathrm{Cr} \bullet 210.3 \mathrm{I}$ & - \\
\hline 76 & $\mathrm{Cr} \bullet 227.1 \mathrm{I}$ & - \\
\hline 8 & $\mathrm{Cr} \bullet 143.8 \mathrm{I}$ & - \\
\hline $8 \mathrm{a}$ & $\mathrm{Cr} \bullet 162.7 \mathrm{I}$ & - \\
\hline 86 & $\mathrm{Cr} \bullet 179.1 \mathrm{I}$ & - \\
\hline 9 & $\mathrm{Cr} \bullet 121.5 \mathrm{I}$ & - \\
\hline $9 \mathrm{a}$ & $\mathrm{Cr} \bullet 123.5 \mathrm{I}$ & - \\
\hline 96 & $\mathrm{Cr} \bullet 131.5 \mathrm{I}$ & $\begin{array}{c}+ \\
\text { (хлф., толуол) }\end{array}$ \\
\hline 10 & $\begin{array}{l}\mathrm{Cr} \bullet 91.7 \mathrm{Mes} \bullet 115.0 \\
\mathrm{I} \bullet 73.4 \mathrm{Mes} \bullet 20.0 \mathrm{G}\end{array}$ & $\begin{array}{c}+ \\
\text { (толуол, бензол, хлф.) }\end{array}$ \\
\hline $10 \mathrm{a}$ & $\mathrm{Cr} \bullet 146.3 \mathrm{I}$ & - \\
\hline 106 & $\mathrm{Cr} \bullet 171.5 \mathrm{I}$ & - \\
\hline 11 & $\mathrm{Cr} \bullet 115.1 \mathrm{I}$ & - \\
\hline $11 \mathrm{a}$ & $\mathrm{Cr} \bullet 140.0 \mathrm{I}$ & - \\
\hline 116 & $\mathrm{Cr} \bullet 108.8 \mathrm{I}$ & - \\
\hline 12 & $\mathrm{Cr} \bullet 145.8 \mathrm{I}$ & - \\
\hline 13 & $\mathrm{Cr} \bullet 142.7 \mathrm{I}$ & - \\
\hline $13 \mathrm{a}$ & $\mathrm{Cr} \bullet 215.3 \mathrm{I}$ & - \\
\hline 136 & $\mathrm{Cr} \bullet 153.7 \mathrm{I}$ & - \\
\hline 14 & $\begin{array}{c}\mathrm{Cr} \bullet 76.1 \mathrm{Mes} \bullet 116.5 \mathrm{I} \\
\mathrm{I} \bullet 110.6 \mathrm{Mes} \bullet 20.0 \mathrm{Mes}\end{array}$ & - \\
\hline $14 \mathrm{a}$ & $\mathrm{Cr} \bullet 174.6 \mathrm{I}$ & - \\
\hline 146 & $\mathrm{Cr} \bullet 133.1 \mathrm{I}$ & - \\
\hline 15 & $\begin{array}{c}\mathrm{Cr} \bullet 79.6 \mathrm{Mes} \bullet 112.3 \mathrm{I} \\
\mathrm{I} \bullet 89.4 \mathrm{Mes} \bullet 38.5 \mathrm{G}\end{array}$ & - \\
\hline 16 & $\mathrm{Cr} \bullet 215.7 \mathrm{I}$ & - \\
\hline 17 & $\mathrm{Cr} \bullet 93.1 \mathrm{I}$ & - \\
\hline
\end{tabular}

Примечание: «+» - соединение является мезоморфным, «-» соединение немезоморфное. $\mathrm{Cr}$ - кристалл; Mes - мезофаза, I - изотроп, G - стеклование. порфиринов 1-6 осуществляли с использованием дипирролилметанов, 5,10,15,20-тетра-мезо-арилзамещенных 7-17 порфиринов - на основе монопиррольной конденсации.

\section{Изучение термотропного и лиотропного} мезоморфизма липофильных ТФП

В работе были изучены жидкокристаллические свойства липофильных порфиринов 1-17 и их металлокомплексов с цинком и кобальтом. В Таблице 3 приведены температуры фазовых переходов для всех соединений, полученные методом поляризационной микроскопии при нагревании и охлаждении. Данным методом было установлено, что соединения 1a, 1б, 2, 3, 4, 10 амфотропны, т.е. проявляют термотропный и лиотропный мезоморфизм, соединения 14 и 15 термотропны, соединения 1a, 16, 2, 3, 4, 10, 15 стеклуются с сохранением текстуры мезофазы.

При изучении жидкокристаллических свойств 5,15-дизамещенных производных ТФП нами было обнаружено, что термотропный мезоморфизм проявляли 5,15-ди(4-тетрадецилоксифенил)-10,20-дифенилпорфирин 2, 5,15-ди(4-гексадецилоксифенил)-10,20-дифенилпорфирин 3 и 5,15-ди(4-октадецил-оксифенил)-10,20-дифенилпорфирин 4. Полученные мезофазы характеризовались негеометрической зернистой текстурой (Рисунок 3). Установление структуры мезофаз требует дополнительных исследований. Увеличение

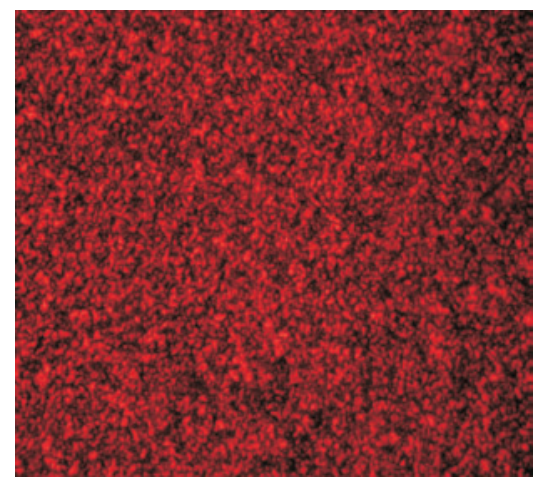

Рисунок 3. Мелкозернистая текстура соединения (3) при нагревании, $\mathrm{T}=61,8^{\circ} \mathrm{C}(\mathrm{Mes}+\mathrm{I})$, поляризаторы скрещены, $\mathrm{x} 250$.

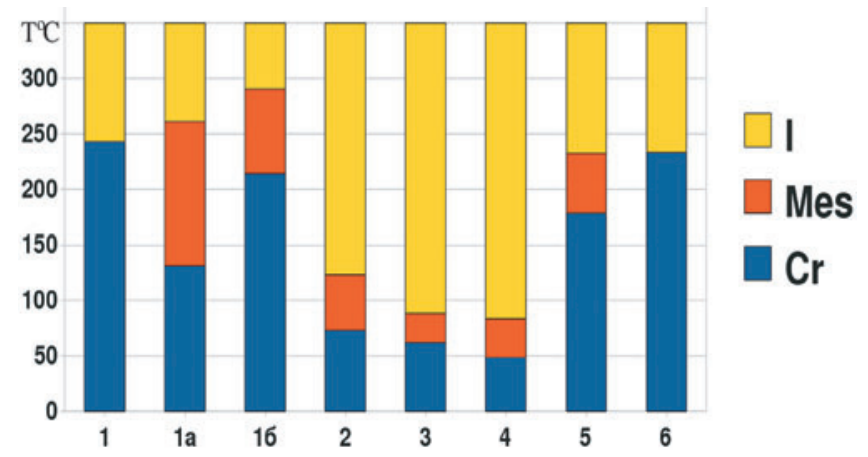

Рисунок 4. Данные по термотропному мезоморфизму 5,15-дизамещенных порфиринов (1-6) по результатам оптической поляризационной микроскопии, $(\mathrm{Cr}$ - кристалл, Mes - мезофаза, I - изотропная жидкость). 
протяженности заместителей понижало температуру фазового перехода кристалл-жидкокристаллическая фаза (Рисунок 4), что согласуется с литературными данными. $^{[3]}$ Порфирин с содержанием в алифатической цепи 8 атомов углерода 5,15-ди(4октилоксифенил)-10,20-дифенилпорфирин 1 не проявил мезоморфных свойств. Однако, важно отметить, что металлокомплексы этого немезоморфного лиганда обладают жидкокристаллическими свойствами: цинковый комплекс 1a проявляет самый широкий интервал существования мезофазы для данной серии порфиринов $\left(129,6^{\circ} \mathrm{C}\right)$, а кобальтовый комплекс $\mathbf{2 a}$ формирует мезофазу в высокотемпературном диапазоне (214,4-290,2 $\left.{ }^{\circ} \mathrm{C}\right)$. Исследование мезоморфных свойств 5,15-дизамещенных порфиринов 5 и 6 с карбоксильными группами показало, что только соединение 5 обладает термотропным мезоморфизмом, формируя мезофазу в интервале температур от $177,5^{\circ} \mathrm{C}$ до $232,5^{\circ} \mathrm{C}$.

Для сравнения мезоморфных свойств ди- и тетразамещенных порфиринов мы использовали 2 серии симметричных тетразамещенных порфиринов 7-11 и 12-17, которые различались способом присоединения периферийных алифатических заместителей к фенильным кольцам ТФП (простая и сложная эфирные связи).

\section{В гомологическом ряду 5,10,15,20-тетра-} (алкоксифенил)порфиринов 7-11 температурный интервал мезофазы $91,7^{\circ} \mathrm{C}-115^{\circ} \mathrm{C}$ наблюдали только для соединения $\mathbf{1 0}$ с длиной периферийного заместителя в 16 углеродных атомов. Соединения 7, 8, 9, 11 оказались немезоморфными, увеличение протяженности заместителей понижало температуру фазового перехода кристалл-изотроп (Рисунок 5).

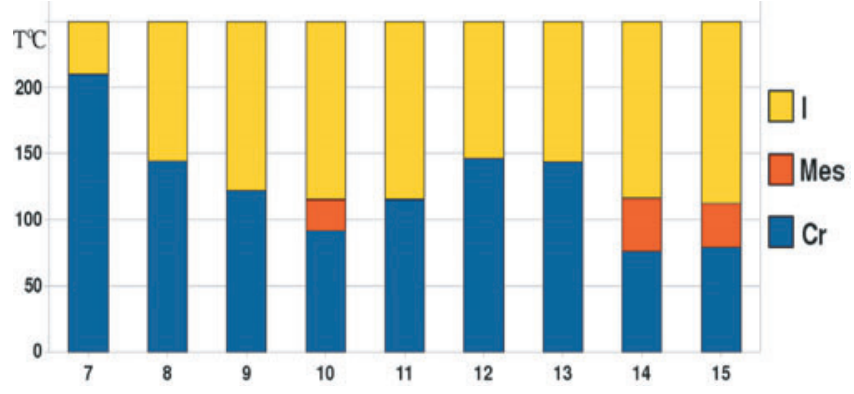

Рисунок 5. Данные по термотропному мезоморфизму 5,10,15,20-тетразамещенных порфиринов по результатам оптической поляризационной микроскопии, $(\mathrm{Cr}$ - кристалл, Mes - мезофаза, I - изотропная жидкость).

В отличие от дизамещенного порфирина 1 нам не удалось индуцировать мезогенные свойства у порфиринов 7, 8, 9, 11 введением координационно ненасыщенных металлов Zn и Со. Вероятно, это связано с различным типом надмолекулярной упаковки у дии тетразамещенных производных порфиринов, что приводит к стерическим затруднениям при формировании молекулярной упорядоченности.

В гомологическом ряду 5,10,15,20-тетра(ацилоксифенил)порфиринов 12-17 были выявлены два мезогенных соединения 14 и 15 с количеством углеродных атомов в периферийных заместителях 14 и 16, соответственно. Температурные интервалы мезофаз этих порфиринов составили $76,1^{\circ} \mathrm{C}-116,5^{\circ} \mathrm{C}$ (14) и $79,6^{\circ} \mathrm{C}-$ $112,3^{\circ} \mathrm{C}$ (15). Для соединения 15 по сравнению с его аналогом 10 температурный переход в мезофазу понижен, и несколько расширен ее интервал. В случае соединения 14 - у его аналога 9 не наблюдается наличия мезофазы. Интересно отметить, что жидкокристаллической фазой не обладали металлокомплексы мезогенных лигандов 14 и 15. По всей видимости, это также связано с разупорядочением структур при экстракоординации металлов макроциклами порфиринов. Соединения 12 и 13 оказались немезоморфными, мезогенные свойства также не удалось индуцировать введением катионов металлов Zn и Co.

Таким образом, мы показали, что изменение природы спейсерного фрагмента и замена электронодонорных алкоксильных заместителей на электроноакцепторные ацилоксильные группы оказывает влияние на мезоморфные свойства производных ТФП, что связано, по-видимому, с большим объемом и электроноакцепторными свойствами карбоксильной группы. Введение терминальных объемных атомов брома в алифатические цепи порфиринов 16 и 17 не приводило к проявлению мезоморфных свойств, что было связано в обоих случаях, вероятно, с недостаточной протяженностью алифатических заместителей.

Следует отметить, что при охлаждении у соединений 1a, 16, 2, 3, 4, 15 происходило стеклование с сохранением текстуры мезофазы (Рисунки 6, 7). В стеклофазе сохраняются анизотропные характеристики предшествующей жидкокристаллической мезофазы, поэтому создание материалов для современных оптических технологий на основе стеклующихся

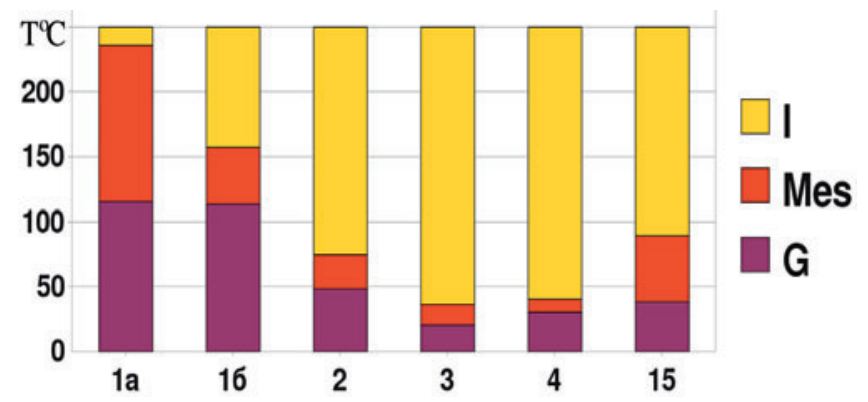

Рисунок 6. Данные по стеклованию липофильных ТФП по результатам оптической поляризационной микроскопии ( $\mathrm{G}$ - стеклование, Mes - мезофаза, I - изотропная жидкость).

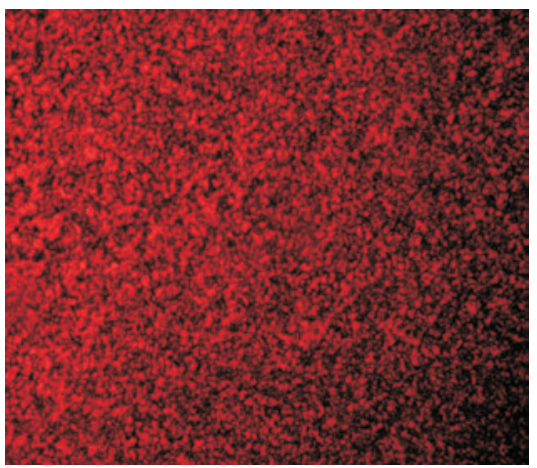

Рисунок 7. Текстура застеклованного состояния соединения 3 при $\mathrm{T}=20,0^{\circ} \mathrm{C}$, поляризаторы скрещены, х250. 


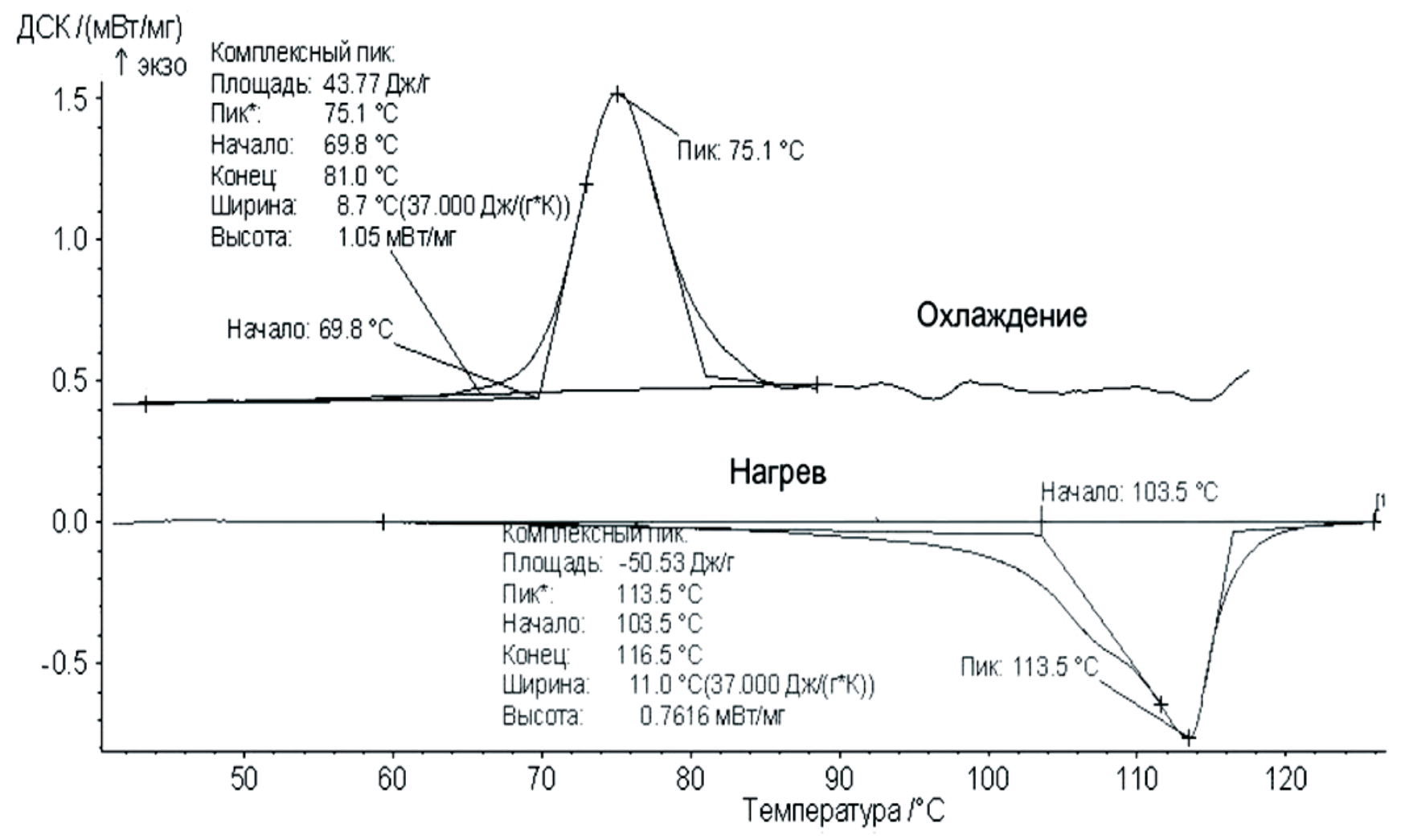

Рисунок 8. Данные ДСК соединения 9 при нагревании и охлаждении.

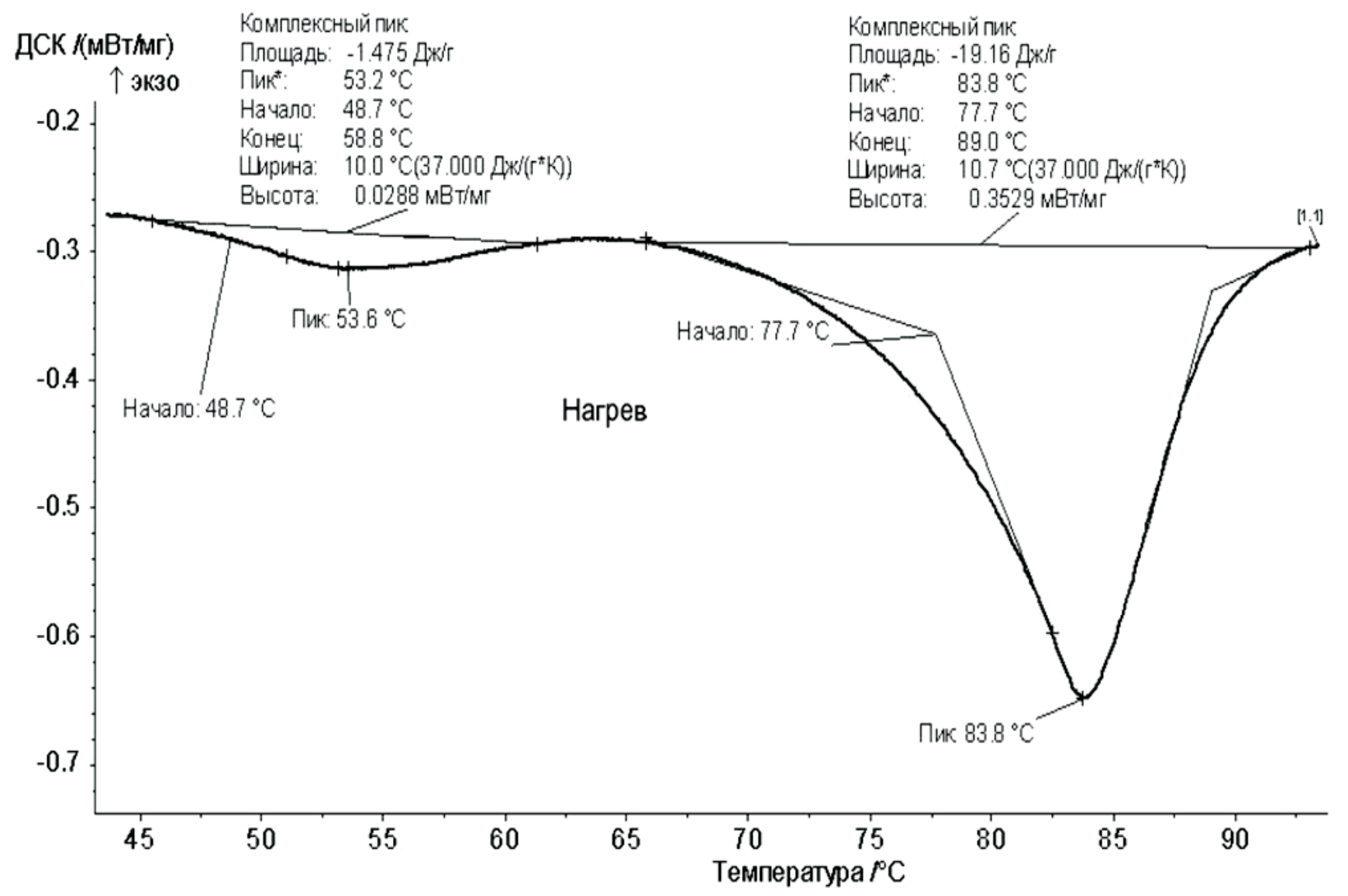

Рисунок 9. Данные ДСК соединения 10 при нагревании. Скорость нагрева $10^{\circ} \mathrm{C} /$ мин. 


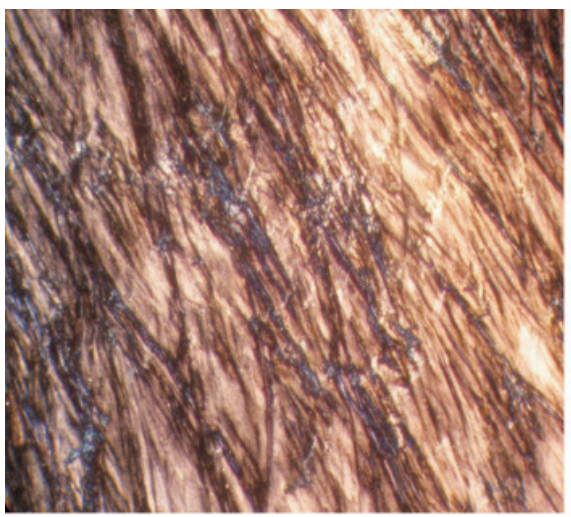

A

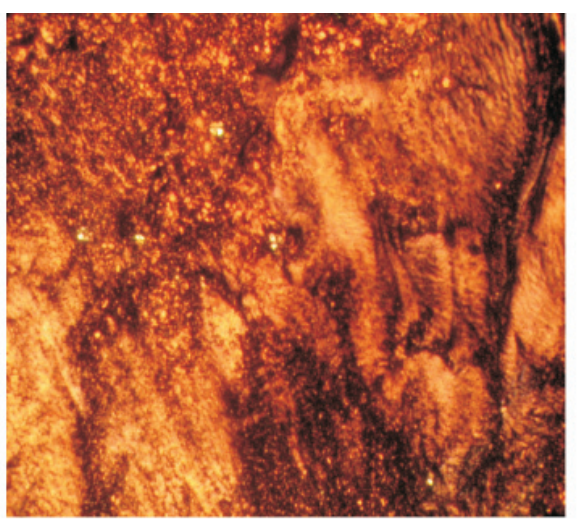

Б

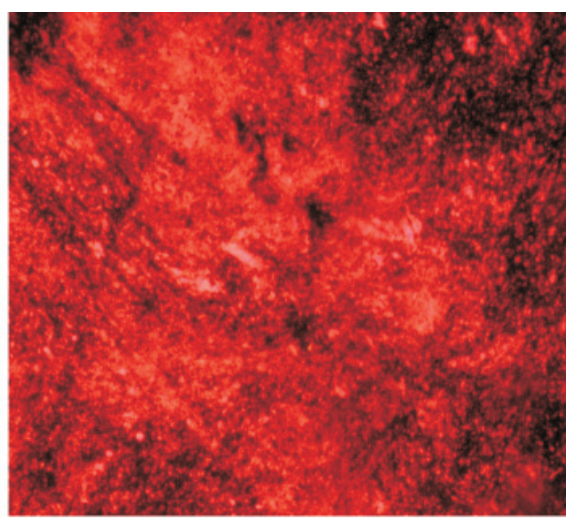

B

Рисунок 10. Текстуры контактных препаратов соединения 3 с толуолом (А), хлороформом (Б) и бензолом (В), поляризаторы скрещены, х250.

мезогенов вызывает особый практический интерес. ${ }^{[5]}$ Однако, фундаментальные представления о причинах стеклования низкомолекулярных органических соединений до сих пор отсутствуют.

Изучение термотропного фазового поведения липофильных тетрафенилпорфиринов методом поляризационной микроскопии было дополнено исследованием с помощью дифференциальной сканирующей калориметрии (ДСК). Для сравнительного анализа были выбраны немезогенное соединение 9 и мезогенное 10. Нагревание проводили в диапазоне температур от 0 до $150^{\circ} \mathrm{C}$. На кривой ДСК соединения 9 отчетливо виден один фазовый переход (Рисунок 8), который согласно данным оптической поляризационной микроскопии соответствует переходу кристалл-изотроп. На кривой ДСК соединения 10 присутствуют несколько фазовых переходов (Рисунок 9). При нагревании этого образца возникают пики, соответствующие двум фазовым переходам кристалл-мезофаза и мезофаза- изотроп.

В результате данной работы также были выявлены порфирины, обладающие лиотропным мезоморфизмом в органических растворителях. Формирование мезофаз в бинарной системе мезоген-растворитель предполагает, с одной стороны, достаточно хорошую растворимость мезогена (все использовавшиеся порфирины обладали этими свойствами), а с другой - сильные межмолекулярные взаимодействия, ответственные за образование надмолекулярных агрегатов - структурных единиц лиотропной жидкокристаллической системы. ${ }^{[28]}$ Изучение методом контактных препаратов с бензолом, толуолом или хлороформом показало, что лиотропным мезоморфизмом обладают соединения 1a, 16, 2, 3, 4, 10 (Рисунок 10).

\section{Выводы}

В результате данного исследования было установлено, что липофильные дискотические производные ТФП проявляют мезоморфные свойства и могут формировать застеклованное состояние при охлаждении. Очевидно, что на жидкокристаллические свойства и стеклообразование порфиринов влияет, прежде всего, количество и протяженность латеральных заместителей, а также способ их присоединения к фенильным остаткам порфирина. Показано, что введение координационно ненасыщенных металлов $\mathrm{Zn}^{\text {II }}$ и $\mathrm{Co}^{\text {II }}$, в целом, увеличивает число мезоморфных гомологов и значительно изменяет мезогенные свойства по сравнению с соответствующими лигандами. При одинаковой длине алифатических заместителей в мезо-положениях макроцикла жидкокристаллические свойства с большей вероятностью будут проявлять 5,15-дизамещенные порфирины и соответствующие металлокомплексы. Таким образом, нами был расширен банк данных ЖК соединений среди липофильных производных порфиринов. Выявлены на основе липофильных производных ТФП термотропные, лиотропные, а также новые стеклующиеся мезогены, которые перспективны в качестве новых материалов для оптоэлектроники.

Благодарности. Работа выполнена при финансовой поддержке РФФИ (грант № 07-03-00427) и АВЦП «Развитие научного потенциала высшей школы» № 2.1.1./2889.

\section{Список литературы}

\section{References}

1. Chou J.H. The Porphyrin Handbook (Kadish K.M., Smith K.M., Guilard R., Eds.), Vol. 6, Amsterdam, Academic Press, 2000, 346 p.

2. Drain C.M, Hupp J.T., Suslick K.S., Wasielewski M.R., Chen X. J. Porphyrins Phthalocyanines 2002, 6, 243-258.

3. Usoltseva N.V., Akopova O.B., Bykova V.V., Smirnova A.I., Pikin S.A. Zhidkiye Kristally - Diskoticheskiye Mezogeny (Liquid Crystals - Discotic Mesogenes) (Usoltseva N.V., Ed.), IGU, Ivanovo, 2004, 128 p. (in Russ.).

4. Eichhorn H. J. Porphyrins. Phthalocyanines 2000, 4, 88-102.

5. Usoltseva N.V., Bykova V.V., Akopova O.B. Uspekhi v Izuchenii Zhidkokristallicheskikh Materialov (Advances in Liquid Crystal Materials), (Usoltseva N.V., Ed.), IGU, Ivanovo, 2007, 100. (in Russ.).

6. Drain C.M. Proc. Natl. Acad. Sci. USA 2002, 99, 5178-5182.

7. Goodby J.W., Robinson P.S., Teo B.K., Cladis P.E. Mol. Cryst. Liq. Cryst. 1980, 56, 303-309.

8. Liu W., Shi Y.H., Shi T.S. Liquid Crystal. 2003, 30, 12551257. 
9. Yu M., Guo. A., Cui X. J. Porphyrins Phthalocyanines 2005, 9, 231-239.

10. Ichihara M., Miida M., Mohr B., Ohta K. J. Porphyrins Phthalocyanines 2006, 10, 1145-1150.

11. Arunkumar C., Bhyrappa P., Varghese B. Tetrahedron Lett. 2006, 47, 8033-8037.

12. Fedulova I.N., Novikov N.V., Bragina N.A., Mironov A.F., Bykova V.V., Usoltseva N.V., Ananieva G.A. Mendeleev Commun. 2008. 18, 324-326.

13. Gregg B.A., Fox M.A, Bard A.J. J. Phys. Chem. 1990, 94, 1586-1598.

14. Schoulten P.G., Warman J.M. Nature 1991, 353, 736.

15. Ohta K., Yamaguchi N., Yamamoto I. J. Mater. Chem. 1998, 8, 2637-2650.

16. Bruce D.W, Wail M.A, Wang Q.M. Chem. Commun. 1994, 18, 2089-2090.

17. Bykova V.V., Usoltseva N.V., Ananieva G.A., Fedulova I.N., Bragina N.A., Mironov A.F. Zhidkiye Kristally $i$ ikh Prakticheskoe Ispolzovaniye (Liquid Crystals and Their Application) 2008, 1, 43-48. (in Russ.).

18. Monobe H., Mima S., Sugini T., Shimizu Y. J. Mater. Chem. 2001, 11, 1383-1392.
19. Yu M., Chen G., Liu G. J. Phys. Chem. Solids 2007, 68, 541548.

20. Monobe H., Miyagama Y., Mima S., Sugini T., Shimizu Y. Thin Solid Films 2001, 393, 217-224.

21. Lee C.-H., Lindsey J.S. Tetrahedron 1994, 50, 11427-11440.

22. Fedulova I.N., Bragina N.A., Mironov A.F. Russ. J. Bioorg. Chem. 2007, 33, 589-593.

23. Lindsey J.S., Hsu H.C., Schreiman I.C. Tetrahedron Lett. 1986, 27, 4969-4970.

24. Fedulova I.N., Novikov N.V., Bragina N.A., Mironov A.F. Vestnik MITCHT 2007, 2, 83-89. (in Russ.).

25. Porfiriny: Struktura, Svoistva, Sintez (Porphyrins: Structure, Properties and Synthesis) (Enikolopyan N.S., Ed.), Nauka, Moscow, 1987, 333 p. (in Russ.).

26. Semeikin A.S., Syrbu S.A., Koifman O.I. Izvestiya Vissh. Ucheb.zaved. (Chimiya i Chimich. Tekhnol.) 2004, 47, 46-55. (in Russ.).

27. Brinas R.P., Bruckner C. Tetrahedron 2002, 58, 4375-4381.

28. Usol'tseva N.V. Liotropnye Zhidkiye Kristally: Khimicheskaya i Nadmolekulyarnaya Struktura (Lyotropic Liquid Crystals: Chemical and Nadmolecular Structure) IGU, Ivanovo, 1994, 220 p. (in Russ.). 